\title{
EFFECT OF INDUCED SUBCLINICAL HYPOCALCEMIA ON THE MOTILITY OF THE BOVINE DIGESTIVE TRACT
}

\author{
Shigeo Hara ${ }^{1}$, Yumi Ikegaya ${ }^{1}$, Rolf Jess Jørgensen ${ }^{2}$, Jyuso Sasaki ${ }^{3}$, Michiko Nakamura $^{1}$ and \\ Nobuyuki Tomizawa ${ }^{1}$ \\ ${ }^{1}$ Department of Veterinary Surgery, and 3 Veterinary Teaching Hospital, \\ Faculty of Agriculture, Iwate University, Morioka 020?8550, Japan. \\ ${ }^{2}$ Department of Clinical Studies, The Royal Veterinary and Agricultural University, \\ DK1870 Frederiksberg C, Denmark
}

\begin{abstract}
The motility of the digestive tract of cows having hypocalcemia induced by administrating Na2EDTA was monitored in three cows through force transducers sutured on peripheral surface of the rumen, body of the abomasum, pylorus, duodenal bulb, descending portion of the duodenum, jejunum and ileum with the purpose to investigate the influence of the concentration of calcium ion $[\mathrm{Ca}+2]$ in plasma on the motility of the digestive tract.

The contraction was decreased in the respective portions of the digestive tract with decreasing $\mathrm{Ca}+2$ concentration in plasma prior to showing anorexia or ataxia observed in milk fever. The rumen was most sensitive to plasma [Ca+2]. When plasma [Ca+2] was decreased to $0.82 \mathrm{mmol} / \mathrm{l}$, contractions began to decrease in amplitude. When the concentration was further decreased to $50 \%$ of the initial value, the contraction only remained $46 \%$ of the initial level. In contrast the descending portion of the duodenum still showed $66 \%$ of the full contraction when the plasma [Ca+2] was $50 \%$ of the initial value. In the abomasum, stronger suppression of the contraction was observed in the pylorus than in the corpus fundus.

When plasma $[\mathrm{Ca}+2]$ was decreased to $50 \%$, the contraction became $61 \%$ of the full in the body, $47 \%$ in the pylorus. During administration of Na2EDTA, phase III of small intestine was often suppressed in the duodenum, jejunum and ileum. Even when phase III was observed the intervals of migrating contractions were prolonged. The results obtained in this study showed that subclinical hypocalcemia brought about suppression of the motility in the respective portions of the digestive tract in cows, and that the sensitivity to hypocalcemia with regard to smooth muscle contraction was different among the portions. This was probably due to the difference in function of the nerves connected to the portions. It was suggested that diminished motility of the rumen and abomasum, caused by subclinical hypocalcemia, was one of the mportant pathogenic factors of enterectacsis or derangement of the abomasum. Additionally, the decreased motility of the small intestine lowered the uptake of $\mathrm{Ca}+2$, and this vicious circle brought about constipation.
\end{abstract}

